\title{
Sorption kinetics for the removal of aldehydes from aqueous streams with extractant impregnated resins
}

\author{
Katarina Babić • Louis G.J. van der Ham • \\ Andre B. de Haan
}

Received: 30 April 2007 / Revised: 7 September 2007 / Accepted: 20 December 2007 / Published online: 23 January 2008

(C) The Author(s) 2008

\begin{abstract}
The sorption kinetics for the removal aldehydes from aqueous solutions with Amberlite XAD-16 and MPP particles impregnated with Primene JM-T was investigated. A model, accounting for the simultaneous mass transfer and chemical reaction, is developed to describe the process. It is based on the analogy to the diffusion and reaction in a stagnant liquid sphere, but corrected for the porosity and particle properties influencing the diffusion. The developed model describes the kinetic behavior of the process in the low concentration region rather well. However, in the high concentration region, larger discrepancies are observed. Initially, the influence of the flow rate was investigated to eliminate the effect of the external mass transfer. The influence of the particle morphology was investigated for both physical and reactive sorption. Physical sorption experiments were used to determine the factor $\tau$ that takes the particle properties influencing the diffusion into account. It was shown that the diffusion is faster in XAD-16 than in MPP impregnated systems. Reaction rate constant $k_{x}$ was determined by fitting the model to the experimental data. Sorption of benzaldehyde appears to be significantly slower $\left(k_{x} \sim 10^{-4} \mathrm{l} / \mathrm{mol} \mathrm{s}\right)$ than the sorption of pentanal $\left(k_{x} \sim 10^{-3} 1 / \mathrm{mol} \mathrm{s}\right)$ due to the slower chemical reaction. The influence of the particle size
\end{abstract}

K. Babić · L.G.J. van der Ham

Faculty of Science and Technology, University of Twente,

P.O. Box 217, 7500 AE Enschede, The Netherlands

Present address.

K. Babić $(\bowtie)$

Sabic Europe, P.O. Box 319, 6160 AH, Geleen, The Netherlands

e-mail: katarina.babic@sabic-europe.com

\section{A.B. de Haan}

Department of Chemical Engineering and Chemistry, Eindhoven

University of Technology, P.O. Box 513, 5600 MB, Eindhoven,

The Netherlands was investigated for the sorption of pentanal with XAD-16. It was observed that the particle size does influence the diffusion term, but does not have an effect on the reaction rate. On the other hand, the extractant loading influences the reaction rate slightly in the low concentration region, whereas the initial concentration of the solute has more pronounced effect.

Keywords Extractant impregnated resins · Kinetics · Zero length column $\cdot$ Aldehydes $\cdot$ Amines

\section{Nomenclature}

$C$ Concentration in liquid phase [mol/l]

$V \quad$ Volume [1]

$t$ Time [s]

$r \quad$ Radial coordinate [m]

$R \quad$ Particle radius [m]

$\phi \quad$ Dimensionless radial coordinate

$\gamma \quad$ Stoichiometric coefficient

$R_{\text {Ald }}$ Reaction rate [mol/ls]

$k \quad$ Reaction rate constant $[1 / \mathrm{mols}]$

$K_{r} \quad$ Equilibrium constant [1/mol]

$K_{p h}$ Physical distribution constant

$\varepsilon_{p} \quad$ Fraction of the EIR particle occupied by the liquid extractant [1 org/l eir]

$D_{i} \quad$ Molecular diffusivity of a component $i\left[\mathrm{~m}^{2} / \mathrm{s}\right]$

$\tau \quad$ Factor encountering for the particle properties influencing the diffusion

$J_{\text {Ald }}$ Flux of the aldehyde through the outer particle surface $\left[\mathrm{mol} / \mathrm{sm}^{2}\right]$

$a_{S} \quad$ Specific surface area $\left[\mathrm{m}^{2} / \mathrm{m}^{3}\right]$

\section{Super and subscripts}

org Organic

$a q$ Aqueous 
$0 \quad$ Initial

$x \quad$ Forward

$-x \quad$ Backward

\section{Abbreviations}

EIR Extractant impregnated resin

Ald Aldehyde

Am Amine

AldAm Schiff base

\section{Introduction}

Extractant Impregnated Resin (EIR) technology (Cortina and Warshawsky 1997) was proposed by us for the removal of aldehydes from aqueous streams, yielding residual concentrations of ppb-level (Babić et al. 2006), either to protect the environment or to obtain a valuable product. Since EIR technology is a synergistic combination of adsorption and reactive extraction, it combines a high capacity and selectivity with relatively simple equipment and operation. The concept of EIRs (Fig. 1) is based on the incorporation of a selective extractive reagent into a porous particle by physical impregnation. When contacted with an aqueous solution the reagent forms a complex with a solute. Both reagent and the formed complex remain in the resin phase as they are insoluble in the aqueous phase.

As complexing agent the primary amine Primene JM-T was used. It shows a reversible yet sufficiently strong reaction with aldehydes, being able to selectively increase the aldehydes affinity for the organic phase by several orders of magnitude. The extraction capability towards benzaldehyde, in both liquid-liquid and impregnated resin systems was tested in our previous work (Babić et al. 2006). In that work three different macroporous adsorbents, Amberlite XAD-16, MPP and Stamypor, were evaluated as solid support.

For the complete understanding of the sorption process, as well as the subsequent equipment and process design, besides equilibrium data, also the sorption kinetics has to be known. Sorption kinetics for extractant impregnated resin systems was mainly investigated assuming the analogy with the ion-exchange resins (Cortina et al. 1998) and described by two frequently used models: the homogeneous particle diffusion model and the shrinking core model (Juang and Lin 1995a, 1995b; Kabay et al. 2003; Saha et al. 2004). Some papers also report more rigorous modeling based on Fick's law of diffusion (Serarols et al. 1999, 2001; Ruiz et al. 2002, 2004; Juang and Lin 1995a, 1995b; Traving and Bart 2002; Kostova et al. 2007) assuming a surface reaction between the solute and the impregnated extractant. In almost all of these kinetics studies, intraparticle diffusion was identified as the rate controlling step.

In our previous study (Babić et al. 2006) a preliminary investigation of the sorption kinetics of benzaldehyde on Primene JM-T impregnated XAD-16 and MPP using a stirred cell was reported. Using the modified shrinking core model (Bhandari et al. 1992) it was shown that the kinetics of sorption is influenced by both intraparticle diffusion and the chemical reaction between the aldehyde and the amine. Up to our knowledge, among all the EIR studies reported in literature, this case was described only by Juang and Lin for the recovery of $\mathrm{Zn}^{2+}$ from aqueous solutions with D2EHPA impregnated Amberlite XAD-2 and XAD-4 (Juang and Lin 1995a, 1995b).

In this paper, we present a more extensive kinetic analysis and develop a model that describes the sorption of aldehydes in more detail. In the case of the sorption of aldehydes in resins impregnated with a primary amine, the reaction takes place in the bulk of the organic amine phase and not at the surface interface of the resin. For derivation of the model it is assumed that the particle is inert and fully impregnated with a reactive liquid. The description of diffusion and reaction is then identical to that of diffusion and reaction in a stagnant liquid sphere taking porosity and other particle

Fig. 1 EIR particle

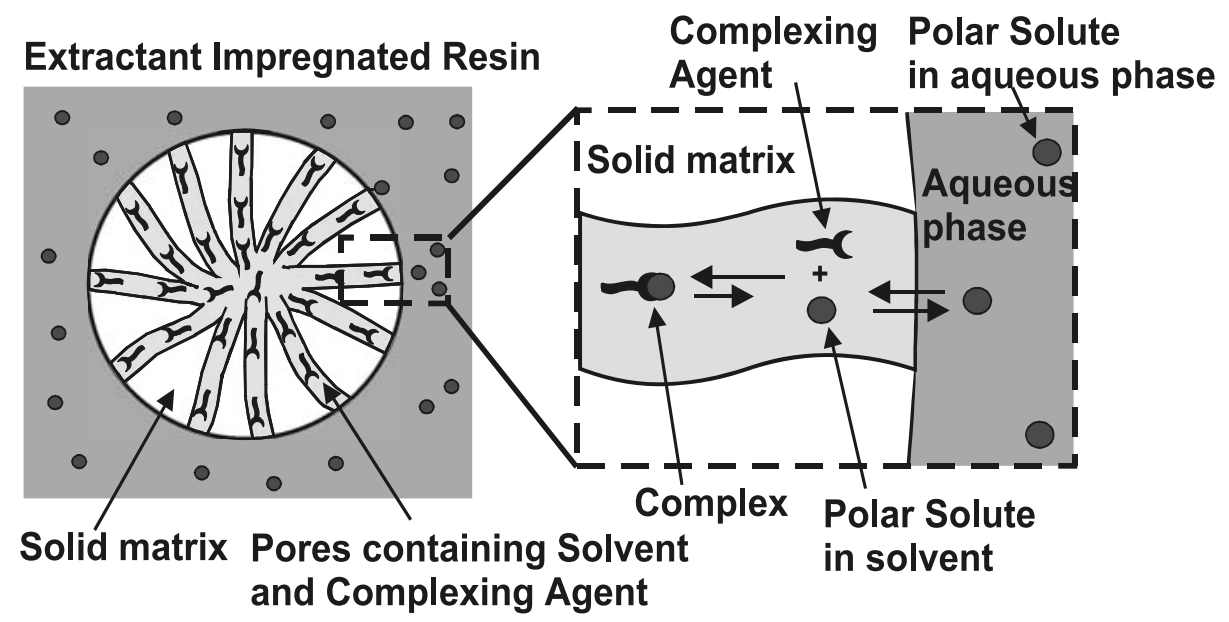


properties (like tortuosity) into account. This concept was previously applied by Hoogendoorn et al. (1993) and evaluated for the absorption of the acid gases $\mathrm{CO}_{2}$ and $\mathrm{H}_{2} \mathrm{~S}$ in alumina particles impregnated with reactive alkanolamine solutions (Hoogendoorn et al. 1994).

The developed model was experimentally evaluated for the sorption kinetics of pentanal and benzaldehyde using the Zero Length Column (ZLC) method (Eić and Ruthven 1988; Ruthven and Stapleton 1993; Dunnewijk et al. 2006). Experiments were performed at sufficiently high flow rates to eliminate the effect of external mass transfer and axial dispersion. Furthermore, the influence of the EIR properties like particle support material, particle size and extractant loading as well as the influence of the molecular structure of the aldehyde and its initial concentration was studied.

\section{Experimental}

\subsection{Materials}

Benzaldehyde and 1-phenyldodecane were purchased from Merck, Germany. Pentanal was obtained from Fluka, Germany. A sample of primary amine Primene ${ }^{\circledR}$ JM-T was received from Rohm and Haas, Europe. All chemicals were used as received. All aqueous solutions were made using MiliQ water. Amberlite XAD-16 was purchased from Sigma Aldrich, Germany. Macroporous polypropylene (MPP) particles were donated by Akzo Nobel, the Netherlands.

Impregnation of the resins was done as described in our previous work (Babić et al. 2006). The extractant loadings of the particles used in this study are listed in Table 1. Impregnated particles were sieved in the following diameter fractions: $0.25-0.5,0.5-0.71,0.71-1.0$ and $>1.0 \mathrm{~mm}$. In all of the experiments, the fraction of $0.71-1.0 \mathrm{~mm}$ was used, except in those experiments where the influence of the particle size was investigated.

\subsection{Experimental set-up and operating procedure}

The ZLC set-up used for the kinetics experiments is shown in Fig. 2. The set-up comprised a glass vessel, a piston pump, an UV/Vis detector and a column packed with a thin layer of EIRs.

For experiments with pentanal $40 \mathrm{ml}$ of aqueous solution of various concentrations $(0.0048 \mathrm{~mol} / \mathrm{l}, 0.011 \mathrm{~mol} / \mathrm{l}$, $0.027 \mathrm{~mol} / \mathrm{l}$ ) was used. Experiments with benzaldehyde were done with $50 \mathrm{ml}$ of aqueous solution with an initial concentration of $0.001 \mathrm{~mol} / \mathrm{l}$.

For adjusting the flow rates a piston pump (Knauer K100 ) was used. The flow was varied from 10 to $40 \mathrm{ml} / \mathrm{min}$. The change in concentration was monitored on-line with a fast-scanning UV/Vis spectrophotometer (Wellchrom/
Table 1 Parameters used in the model calculations

Model parameters

\begin{tabular}{lllll}
\hline Aldehyde & & & Pentanal & Benzaldehyde \\
\hline$K_{r}$ & $1 / \mathrm{mol}$ & & 200 & 168 \\
$K_{p h}$ & $l_{\text {org }} / l_{a q}$ & & 15.8 & 14.8 \\
$D \times 10^{6}$ & $\mathrm{~cm}^{2} / \mathrm{s}$ & in JM-T & 1.74 & 1.74 \\
& & in 1PD & 2.76 & -
\end{tabular}

Extractant properties

\begin{tabular}{llll}
\hline & & Primene JM-T & 1-phenyldodecane \\
\hline Molar mass & $\mathrm{g} / \mathrm{mol}$ & 269 & 246.4 \\
Density & $\mathrm{g} / \mathrm{ml}$ & 0.834 & 0.850 \\
Viscosity & $\mathrm{cP}$ & 11.59 & 7.0
\end{tabular}

Particle properties

\begin{tabular}{lllll}
\hline Resin & Extractant & $\begin{array}{l}\text { Loading } \\
\mathrm{mol} / 1_{\text {EIR }}\end{array}$ & $\begin{array}{l}\varepsilon_{p} \\
-\end{array}$ & $\begin{array}{l}\text { Density } \\
\mathrm{g} / \mathrm{ml}_{\text {EIR }}\end{array}$ \\
\hline \multirow{2}{*}{ XAD16 } & 1-PD & 2.51 & 0.7254 & 0.9694 \\
& JM-T & 1.83 & 0.5806 & 1.0079 \\
& JM-T & 2.30 & 0.7346 & 0.9667 \\
MPP & 1-PD & 2.02 & 0.5844 & 0.9862 \\
& JM-T & 1.35 & 0.4379 & 1.0441 \\
& JM-T & 1.85 & 0.5945 & 0.9932 \\
\hline
\end{tabular}

Knauer K-2600) equipped with a $2 \mu$ flow cell. Pentanal was detected at $249 \mathrm{~nm}$ and benzaldehyde at $283 \mathrm{~nm}$.

EIR particles were packed in a $15 \mathrm{~mm}$ diameter column (Omnifit, UK). The volume of the impregnated particles was $0.21 \mathrm{ml}$ in all experiments yielding a packed layer thickness of approximately $2 \mathrm{~mm}$. To assure a constant temperature throughout the experiment the column was placed in an oven. All the experiments were done at $25^{\circ} \mathrm{C}$ (similar to the equilibrium experiments in our previous work (Babić et al. 2006, 2008).

The ZLC set-up can be operated in recycle and nonrecycle mode. Before each experiment, the set-up was equilibrated with pure water. Then the feed tube was placed in the vessel containing the aqueous solution of an aldehyde. As a consequence, the UV detector detected an increase in the sorbate concentration. The feed solution was well mixed to assure homogeneous concentration (simulating a perfectly mixed system).

\subsubsection{Calibration of the set-up}

Prior to the experiments, the set-up needs to be calibrated (Dunnewijk et al. 2006). The volume of the total set-up was determined to be able to calculate the dilution of the initial solution caused by the residual solvent present in the system, i.e. volume of all tubing, pump cell and UV/Vis detector cell 
Fig. 2 ZLC set-up

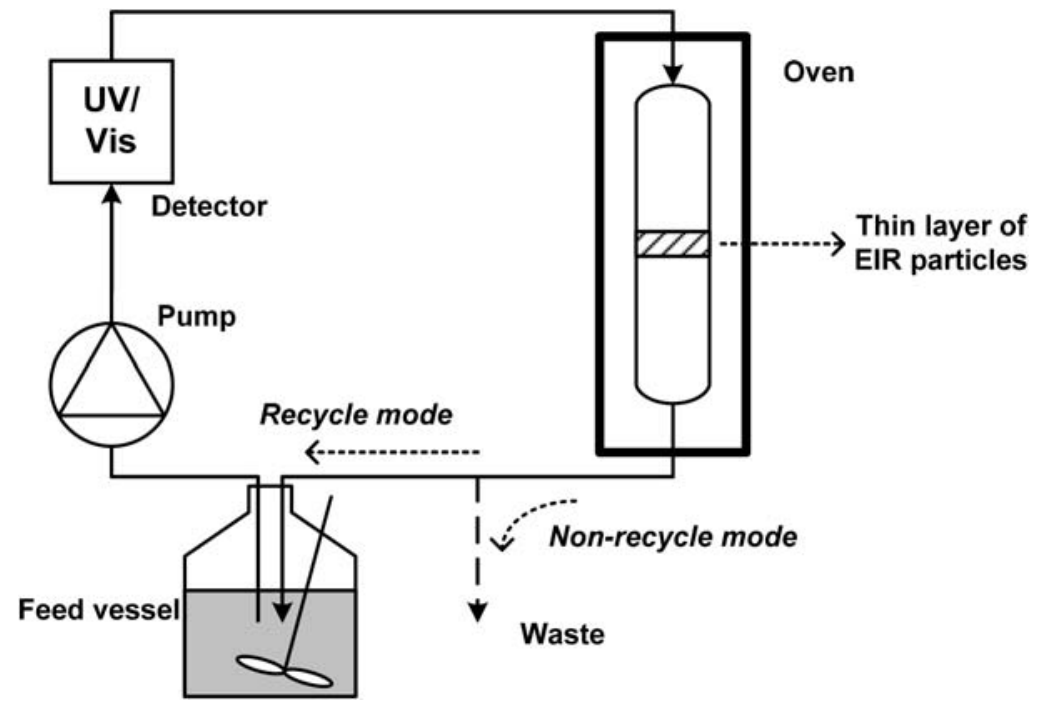

as well as the volume of the porous part of the bed. In all experiments, the bed porosity was 0.41 (calculated as one minus the ratio of the volume of the EIRs present and the total volume of the column). The system volume was calculated from the difference in aldehyde concentration measured in the set-up with an empty bed in both recycle and non-recycle mode. The typical blank response curves can be found in the work of Dunnewijk et al. (2006). For the set-up used in these experiments, it was determined that this volume is $3.2 \pm 0.1 \mathrm{ml}$.

\section{Mathematical model}

\subsection{Model description}

When a particle impregnated with Primene JM-T is contacted with an aqueous solution of aldehyde, the aldehyde molecules first dissolve in the organic liquid where they subsequently react with the amine forming a Schiff base according to the reaction

$\mathrm{R}_{1} \mathrm{R}_{2} \mathrm{CO}+\mathrm{R}_{3} \mathrm{NH}_{2} \underset{k_{-x}}{\stackrel{k_{x}}{\rightleftharpoons}} \mathrm{R}_{1} \mathrm{R}_{2} \mathrm{C}=\mathrm{NR}_{3}+\mathrm{H}_{2} \mathrm{O}$.

For convenience we will proceed with using the following abbreviations as subscripts: Ald for the aldehyde $\left(\mathrm{R}_{1} \mathrm{R}_{2} \mathrm{CO}\right)$, $A m$ for the amine $\left(\mathrm{R}_{3} \mathrm{NH}_{2}\right)$ and AldAm for the Schiff base complex $\left(\mathrm{R}_{1} \mathrm{R}_{2} \mathrm{C}=\mathrm{NR}_{3}\right)$. When first order in the reactants is assumed, the reaction rate becomes

$$
\begin{aligned}
R_{A l d} & =k_{x} C_{A l d}^{\text {org }} C_{A m}^{\text {org }}-k_{-x} C_{A l d A m}^{\text {org }} \\
& =k_{x}\left(C_{A l d}^{\text {org }} C_{A m}^{\text {org }}-\frac{1}{K_{r}} C_{\text {AldAm }}^{\text {org }}\right),
\end{aligned}
$$

where the superscript org refers to the organic phase and the equilibrium constant is given by

$K_{r}=\frac{k_{x}}{k_{-x}}=\frac{C_{A l d A m}^{\text {org }}}{C_{A l d}^{\text {org }} \cdot C_{A m}^{\text {org }}}$.

It is assumed that the particle is inert and spherical. In that case the analogy with the stagnant liquid sphere (droplet) can be applied. Simultaneous mass transfer and chemical reaction in a small sphere should be non-stationary because of the finite capacity of the droplet. The non-stationary mass transfer accompanied by a chemical reaction can be described, for each component in the EIR, by the following mass balance (Hoogendoorn et al. 1993)

$$
\begin{gathered}
\varepsilon_{p} \frac{\partial C_{i}^{\text {org }}}{\partial t}=\varepsilon_{p} \frac{D_{i}}{\tau} \frac{1}{r^{2}} \frac{\partial}{\partial r}\left(r^{2} \frac{\partial C_{i}^{\text {org }}}{\partial r}\right)-\varepsilon_{p} \gamma_{i} R_{A}, \\
i=\text { Ald, Am, AldAm, }
\end{gathered}
$$

where the stoichiometric coefficient $\gamma$ is 1 for Ald and Am and -1 for AldAm. $\varepsilon_{p}$ is the fraction of the EIR particle occupied by the liquid extractant

$\varepsilon_{p}=\frac{V_{\text {org }}}{V_{E I R}}$

and $\tau$ is the correction factor encountering for the particle properties influencing the diffusion (like tortuosity). The decrease of the aldehyde concentration in the aqueous phase can be calculated from the following mass balance

$V_{a q} \frac{\partial C_{A l d}^{a q}}{\partial t}=-a_{S} V_{E I R} J_{A l d}$,

where $J_{A l d}$ is the flux of the aldehyde through the outer particle surface. The superscript $a q$ refers to the aqueous phase. 
Since the reaction between aldehyde and amine is of a finite rate, the flux is given by

$J_{A l d}=\left.\varepsilon_{p} \frac{D_{A l d}}{\tau} \frac{\partial C_{A l d}^{\text {org }}}{\partial r}\right|_{r=R}$.

The specific surface area as for spherical particles equals

$a_{S}=\frac{3}{R}$.

To improve the scalability of the model a dimensionless variable for the radius is introduced

$\varphi=\frac{r}{R}$.

Combining (2), (4), (6)-(9), the model equations can finally be written as follows. EIR phase:

$$
\begin{aligned}
& \varepsilon_{p} \frac{\partial C_{i}^{\text {org }}}{\partial t}= \varepsilon_{p} \frac{D_{i}}{\tau R^{2}} \frac{1}{\varphi^{2}} \frac{\partial}{\partial \varphi}\left(\varphi^{2} \frac{\partial C_{i}^{\text {org }}}{\partial \varphi}\right) \\
&-\varepsilon_{p} \gamma_{i} k_{x}\left(C_{\text {Ald }}^{\text {org }} C_{A m}^{\text {org }}-\frac{1}{K_{r}} C_{\text {AldAm }}^{\text {org }}\right), \\
& i=\text { Ald }, \text { Am }, \text { AldAm. }
\end{aligned}
$$

Aqueous phase:

$$
\frac{\partial C_{A l d}^{\text {org }}}{\partial t}=-\left.3 \varepsilon_{p} \frac{D_{\text {Ald }}}{\tau R^{2}} \frac{V_{E I R}}{V_{a q}} \frac{\partial C_{A l d}^{\text {org }}}{\partial \varphi}\right|_{\varphi=1} .
$$

Since there is a clear physical solubility of aldehydes in the organic phase, it is not likely that the chemical reaction will be restricted to the interface. It is to be expected that it takes place in the bulk of the organic phase. However, at the interface, it can be assumed that there is a physical equilibrium of the solute. Therefore, following boundary condition can be written

$\left.C_{A l d}^{\text {org }}\right|_{\varphi=1}=K_{p h} C_{A l d}^{a q}$,

where $K_{p h}$ is the physical distribution constant of the aldehyde.

Both the amine and the formed complex are not soluble in the aqueous phase and we assume that it is not likely that they will diffuse out of the particle. Therefore, it can be stated

$\left.\frac{\partial C_{i}^{\text {org }}}{\partial \varphi}\right|_{\varphi=1}=0, \quad i=A m$, AldAm.

Because of the process symmetry

$\left.\frac{\partial C_{i}^{\text {org }}}{\partial \varphi}\right|_{\varphi=0}=0, \quad i=A l d$, Am, AldAm.
In general, initial conditions can be written as follows

$C_{i, 0}^{\text {org }}=$ constant $, \quad \varphi \in[0,1], \quad i=$ Ald, Am, AldAm,

$C_{\text {Ald }, 0}^{a q}=$ constant.

For the experiments done in this work, the initial concentration of the aldehyde and the Schiff base in the organic phase $C_{i, 0}^{\text {org }}$ was zero. Thus, initially the amine is present in the particle in the pure form $\left(C_{A m, 0}^{\text {org }}=3.1 \mathrm{~mol} / \mathrm{l}\right)$.

\subsection{Model parameters}

To successfully solve this model, the values of the model parameters need to be known. The equilibrium constants as well as physical distribution ratios were measured in our previous work (Babić et al. 2008). Diffusivity of aldehydes in Primene ${ }^{\circledR} \mathrm{JM}-\mathrm{T}$ in the lower concentration region was estimated using the Wilke-Chang correlation (Reid et al. 1988) combined with the Le Bas (Reid et al. 1988) contribution method for estimation of the molar volume at boiling point. Compared to the small aldehyde, the diffusion of both the bulky amine and the formed complex was assumed to be negligible and therefore corresponding diffusivities were set to zero. Values of the parameters as well as the initial conditions used in the calculations are listed in the Table 1. Since no study of the reaction kinetics for the reaction of the aldehydes with Primene JM-T was reported in literature, the reaction rate constant had to be estimated by fitting the model to the experimental data. Factor $\tau$ was determined from independent physical diffusion experiments, also by fitting the model (without reaction terms) to the experimental data. It is important to mention that the volume of the aqueous phase $V_{a q}$ was corrected for the system volume. Also, the initial concentration of the solution was calculated taking into account the dilution caused by this residual volume.

\subsection{Parameter estimation procedure}

The model was implemented in the computer program gPROMS $^{\circledR}$ (2007) and the difference between experimentally measured data and model was minimized to obtain the optimum value for the reaction rate constant $k_{x}$. The fitting was done with the parameter estimation subroutine within gPROMS $^{\circledR}$ using the maximum likelihood estimator and a heteroscedastic variance model of the measured values (gPROMS $^{\circledR}$ 2007).

\section{Results and discussion}

\subsection{Influence of the flow rate}

Investigation of the intraparticle phenomena can be done if the experiments are conducted in such a way that the ex- 


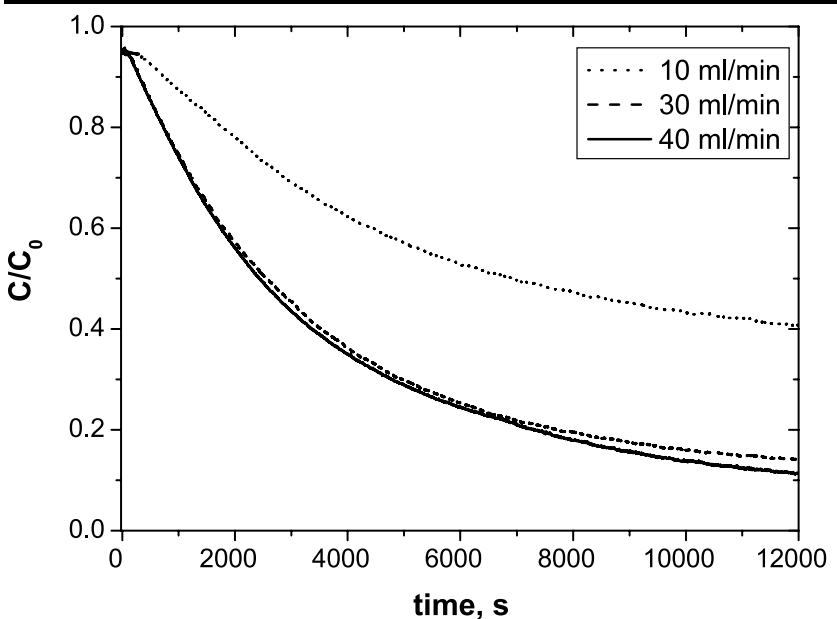

Fig. 3 Influence of the flow rate on the pentanal sorption on XAD-16 impregnated with Primene JM-T (particle size 0.71-1.0 mm)

ternal mass transfer can be neglected. With the ZLC set-up this can be achieved by using sufficiently high flow rates. For the selection of the proper experimental conditions it is important to determine the influence of the flow rate on the sorption rate. Therefore the flows of $10-40 \mathrm{ml} / \mathrm{min}$ were applied. The results for the pentanal sorption on XAD-16 impregnated with Primene JM-T (particle size 0.71-1 mm) are presented in Fig. 3, where it is seen that for flow rates higher than $30 \mathrm{ml} / \mathrm{min}$ the external mass transfer is negligible. The same effect of the flow rate was observed for all other systems investigated in this study (not shown). For this reason all subsequent experiments were done at flow rates of $40 \mathrm{ml} / \mathrm{min}$.

\subsection{Influence of the material}

\subsubsection{Physical sorption system (particles impregnated with non-reactive liquid)}

The influence of the polymeric support is expressed by the parameter $\tau$ in the mathematical model. To determine $\tau$ independent from the complexation reaction, particles were impregnated with a non-reactive liquid (1-phenyldodecane). The mathematical expression of the physical sorption is the same as for the reactive (9)-(15) except for the "reaction terms". By fitting the model to the experimental data the value of $\tau$ was determined to be $3.03 \pm 0.55$ for XAD-16 impregnated systems and $6.51 \pm 0.50$ for MPP impregnated systems. Although, the standard deviation of the model from the experimental results was not exceeding $2.5 \%$, the standard deviation of the experimental measurements is rather high (about 10\%). This is due to the relatively low physical solubility of pentanal in 1-phenyldodecane. Subsequently the change in concentration between the initial and the equilibrium value is rather small for more accurate detection by the UV detector used in this research.
Considering only the particle morphology (pore sizes and pore size distribution), the difference in obtained values for $\tau$ is surprising. However, XAD-16 is somewhat less hydrophobic than MPP which most probably promotes the diffusion of polar organic like aldehyde (Komiyama and Smith 1974). Moreover, it should be noted that XAD-16 is a spherical and MPP a cylindrical particle. Nevertheless, from the work of Ma and Evans (1968) it follows that the cylinders with a length to diameter ratio of about 1 can be approximated by a sphere with an identical radius. For MPP particles used in this research this condition is satisfied. When differences between XAD-16 and MPP are discussed, it should also be noted that even within the same particle size fraction, the particle size distribution was not the same. For the experiments, a fraction of $0.71-1.0 \mathrm{~mm}$ was used, but for XAD-16 the average particle size was $0.8 \mathrm{~mm}$, where for MPP it was $0.95 \mathrm{~mm}$.

\subsubsection{Reactive sorption system (particles impregnated with Primene JM-T)}

Experimental results for the sorption of pentanal with resins impregnated with Primene JM-T are presented in Fig. 4. It can be seen that the sorption is faster for XAD-16 impregnated systems. Fitting the model to the experimental results revealed that the reaction rate constant is the same (within the error of estimation) for both XAD-16 and MPP impregnated systems (Table 2). Therefore, it can be concluded that the difference in sorption rates comes from the particle properties (correlated by the factor $\tau$ ).

\subsection{Influence of the particle size}

The influence of the particle size on the sorption of pentanal was investigated with fully impregnated particles XAD16JMT-2.30. The experimental results as well as the model predictions are presented in Fig. 5. Unlike the previous paragraph, where the value of parameter $k_{x}$ was determined by fitting to the experimental data, here the already determined value (Table 2) was used for simulation of the kinetics behavior. From Fig. 5 it is evident that the model gives rater good predictions of the sorption kinetics for all the particle sizes studied. The model deviation from the experimentally measured data is not more than $5 \%$.

Since all the parameters are identical for all the particle sizes, it can be concluded that there is no influence on the effective diffusion coefficient $\left(\varepsilon_{p} D_{\text {Ald }} / \tau\right)$. However, since the diffusion term (diffusion time constant) is inversely proportional to $R^{2}$, the diffusion is subsequently faster in smaller particles. 


\subsection{Influence of the extractant loading}

The effect of the Primene JM-T loading is presented in Fig. 6. It can be noticed that the sorption rate slightly increases with the increase of the extractant loading (and thus, the volume of the organic phase). When the volume of the organic phase influences the reaction rate, the chemical reaction is slow and occurs in the bulk organic phase (Do-

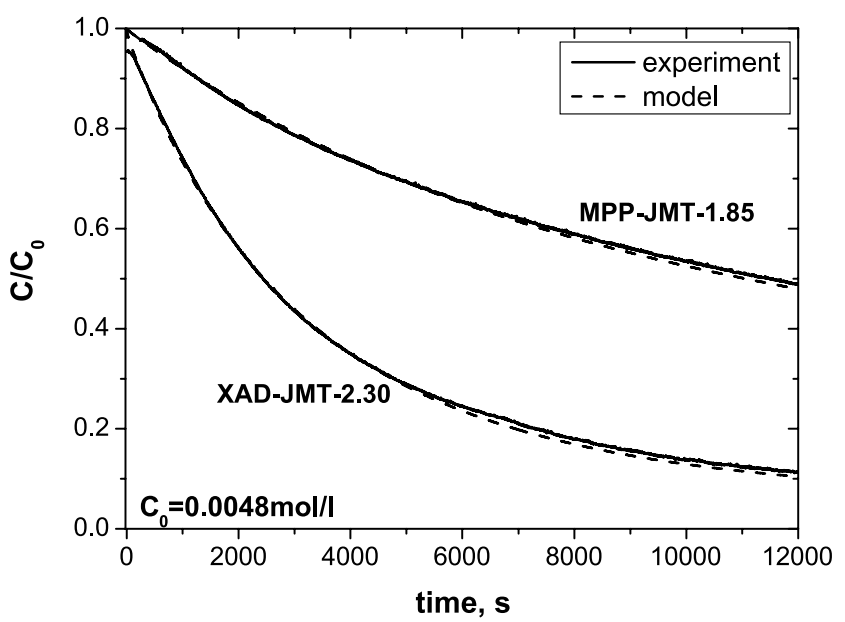

(a)

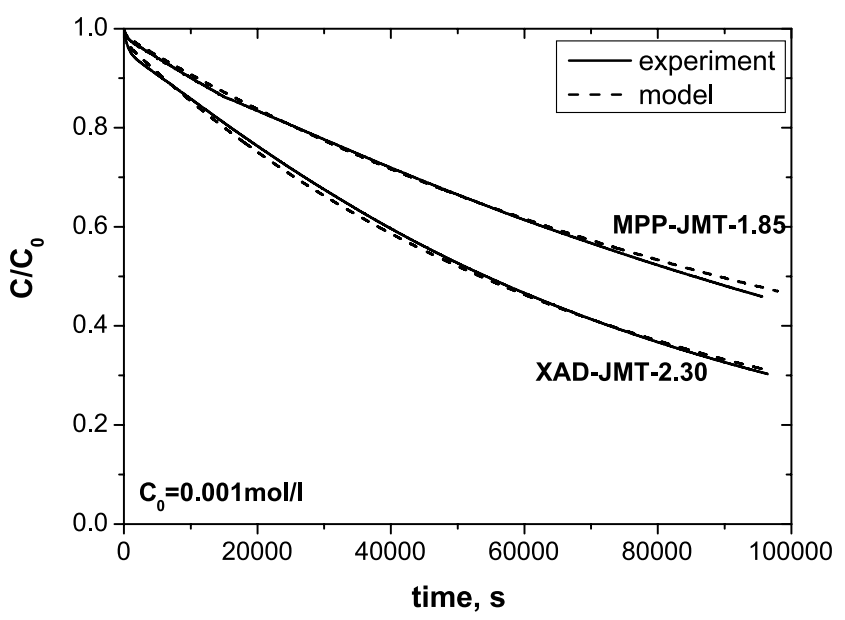

(b)

Fig. 4 Influence of the particle material on the sorption of (a) pentanal and (b) benzaldehyde raiswamy and Sharma 1984; Nikhade and Pangarkar 2005). This confirms the justification of the initial model assumption. Increased loading of the extractant means an increased value of the parameter $\varepsilon_{p}$ and from (10) it follows that the decrease in aqueous concentration of aldehyde will be larger. However, for the low aldehyde concentrations this effect is not so pronounced since there is an excess of amine for the reaction.

\subsection{Influence of the solute's initial concentration}

The influence of the initial concentration of aldehydes was investigated for both XAD16 and MPP fully impregnated systems. It is known from literature (Lee 2004; Nikhade and Pangarkar 2005) that the higher the initial concentration the faster the reaction rate. After the chemical equilibrium is reached the diffusion becomes the only limiting resistance. The experimental results as well as model predictions are presented in Fig. 7. A large discrepancy between the model prediction and the experimental values can be observed for the high solute's concentration. Even though the initial sorption rate is well predicted, when approaching the equilibrium, the model starts deviating from the experimental data. It is observed that the equilibrium value differs significantly from our previous work (Babić et al. 2008). It is possible

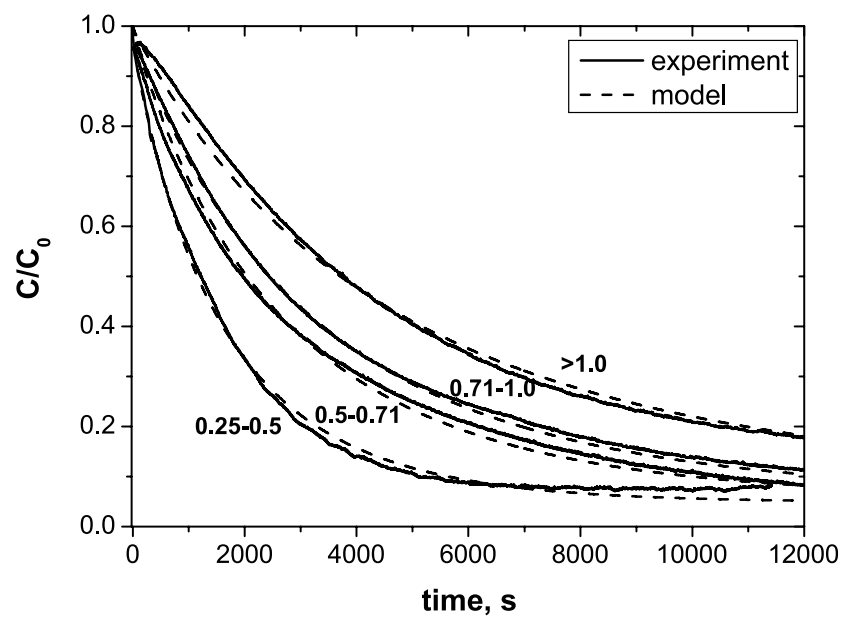

Fig. 5 Influence of the particle size for the XAD16-JMT-2.3 EIR (particle sizes: $0.25-0.5 ; 0.5-0.71 ; 0.71-1.0 ;>1.0 \mathrm{~mm}$ ) and initial pentanal concentration of $0.0048 \mathrm{~mol} / \mathrm{l}$
Table 2 Determined reaction rate constants

\begin{tabular}{llll} 
Reaction rate constant & & \\
\hline Aldehyde & Particle & $\begin{array}{l}\text { Reaction rate constant } \\
k_{x} \times 10^{3} 1(\mathrm{~mol} \mathrm{~s})^{-1}\end{array}$ & $\begin{array}{l}\text { Standard deviation } \\
\sigma^{2} \times 10^{6} 1(\mathrm{~mol} \mathrm{~s})^{-1}\end{array}$ \\
\hline Pentanal & XAD16 & 4.431 & 8.250 \\
Benzaldehyde & MPP & 4.130 & 9.851 \\
& XAD16 & 0.151 & 0.040 \\
\hline
\end{tabular}




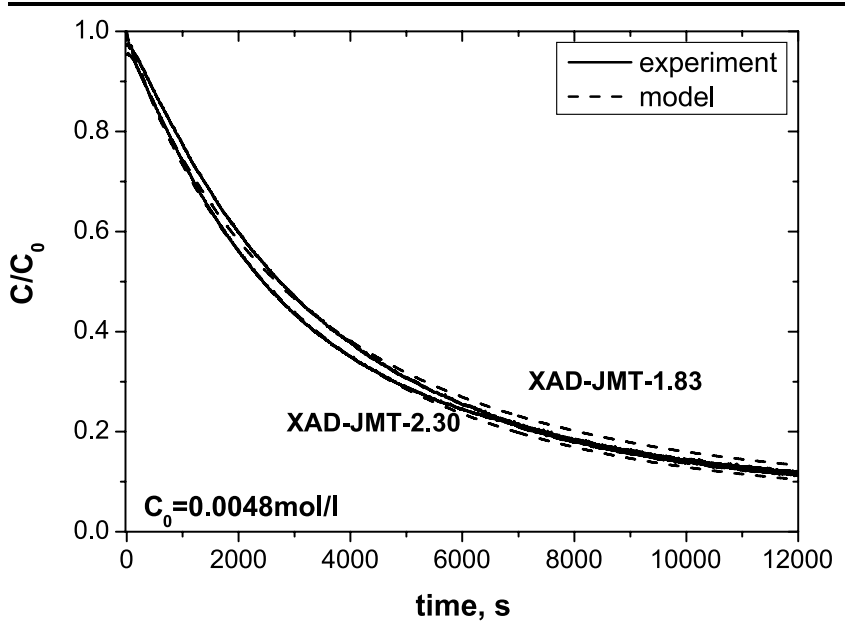

(a)

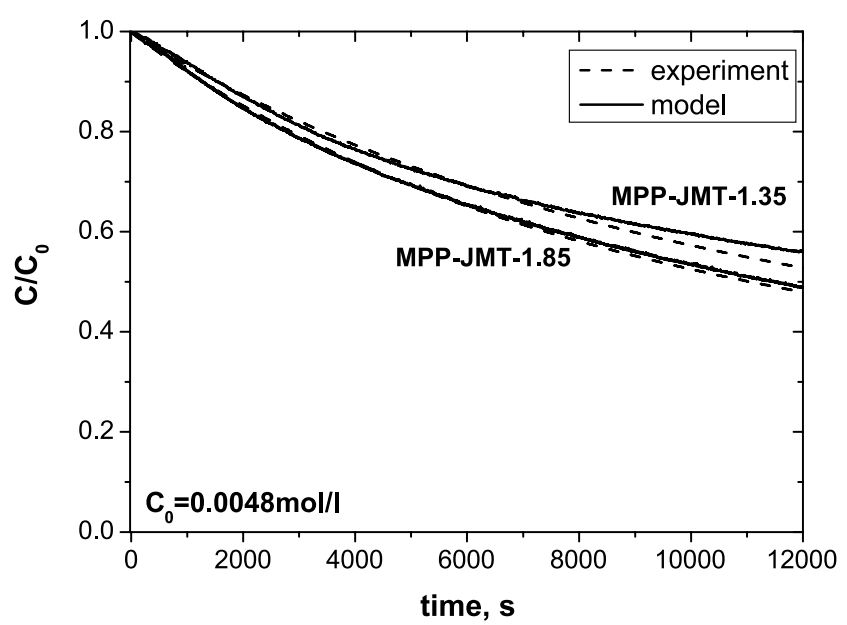

(b)

Fig. 6 Influence of the extractant loading on the sorption of pentanal (initial concentration $0.0048 \mathrm{~mol} / \mathrm{l}$, particle size $0.71-1.0 \mathrm{~mm}$ ): polymeric support (a) XAD-16 (b) MPP

that this is due to the extractant loss during the experiment. This most likely happens as a consequence of the organic phase swelling which influences the immobilization of the extractant. On the other hand, it is possible that the effective diffusivity is lowered due to the increased concentration of the solute and complex inside the pores. The concentration dependency of the effective diffusivity is often taken into account using Darken correction. This effect is also observed for the sorption of phenylglycine on tricaprylylmethylammonium chloride impregnated XAD resins by Kostova et al. (2007).

In Fig. 8 the experimental data of the benzaldehyde sorption measured in a stirred vessel, reproduced from our previous work (Babić et al. 2006), are presented together with the predictions based on the model described here. The initial concentration of benzaldehyde was $0.033 \mathrm{~mol} / \mathrm{l}$. Compared to the results presented in Fig. 4(b), it can be noticed that the initial sorption rate is higher at the higher initial concen-

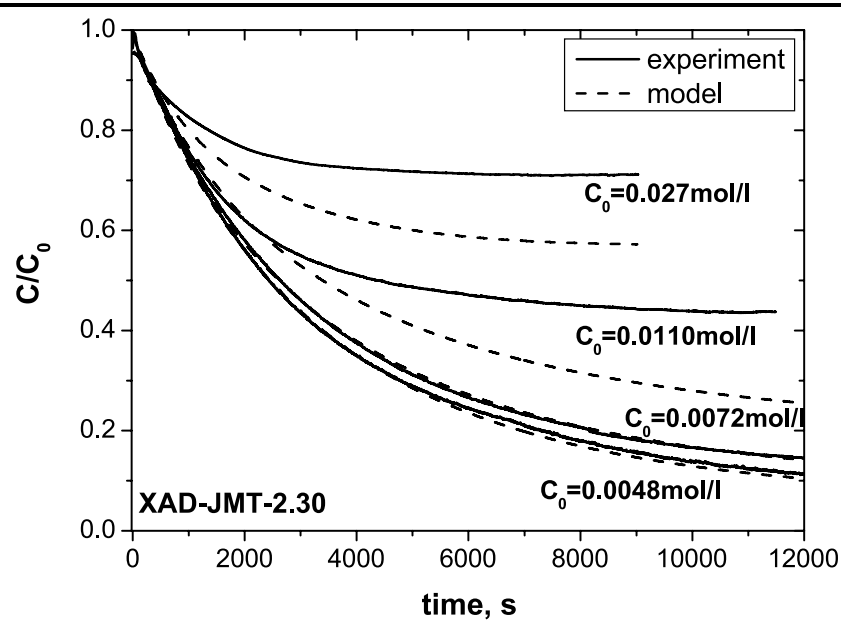

(a)

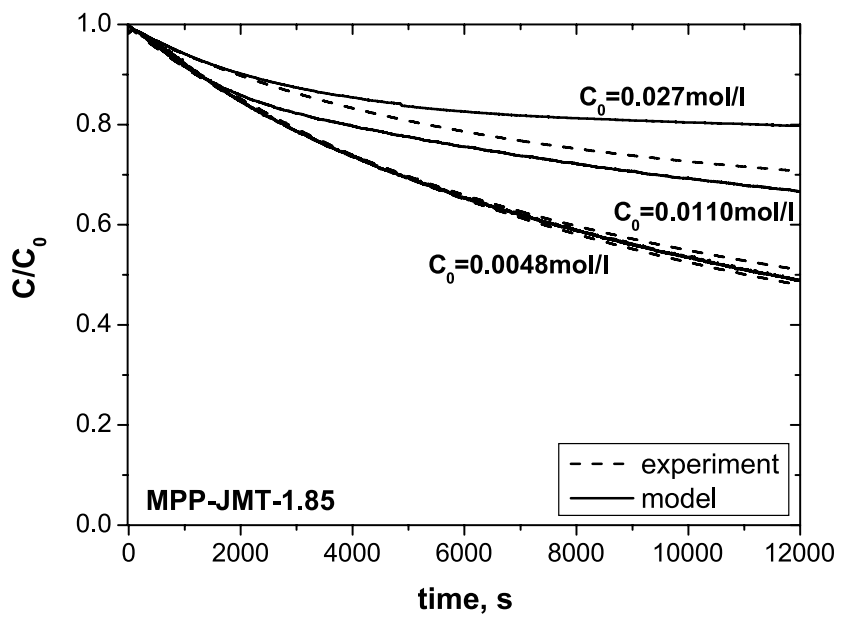

(b)

Fig. 7 Influence of the initial concentration of pentanal: polymeric support (a) XAD16 (b) MPP

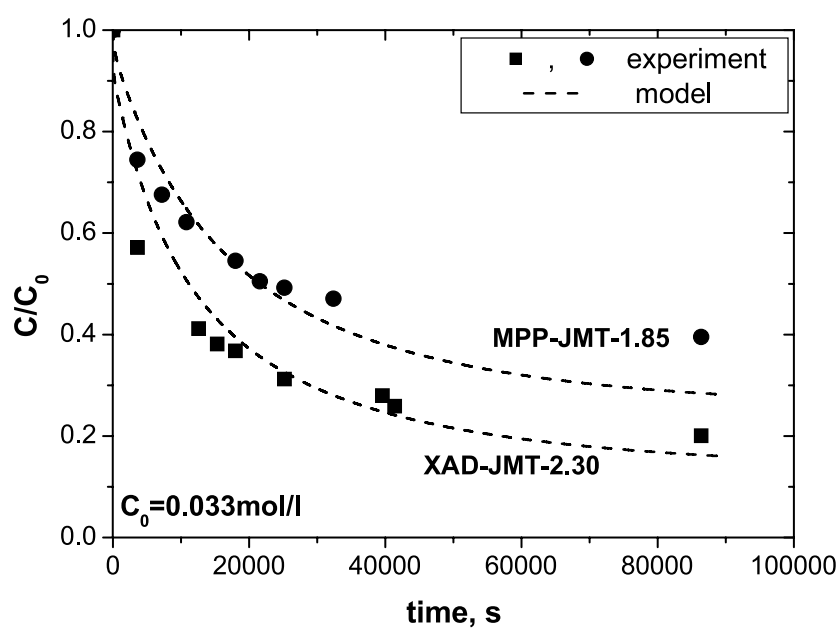

Fig. 8 Sorption of benzaldehyde (initial concentration $0.033 \mathrm{~mol} / \mathrm{l}$ ): points — data from the stirred vessel measurements (Babić et al. 2006), line-model 


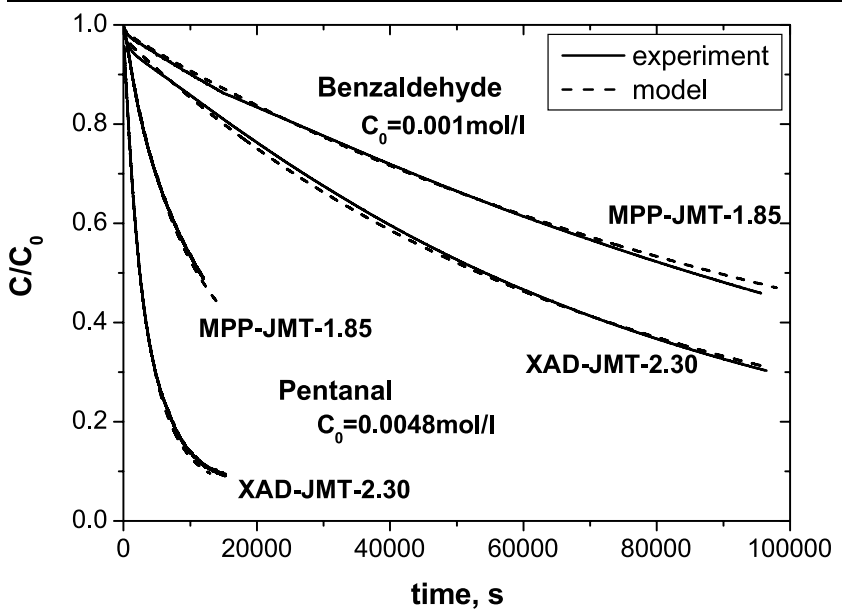

Fig. 9 Comparison of the sorption kinetics of benzaldehyde and pentanal with particles fully impregnated with Primene JM-T (particle size fraction $0.71-1.0 \mathrm{~mm}$ )

tration. Similar was observed by Lee (2004) for the reactive extraction of penicillin G by Amberlite LA-2, as well as by Nikhade and Pangarkar (2005) for the extraction of citric acid with Alamine 336. As well as for the sorption of pentanal at the higher concentrations, the discrepancy between the predicted and measured values is observed. Since the instability of the EIRs has a serious consequence on the future industrial application, further research should provide more insight to this problem.

\subsection{Benzaldehyde sorption}

Experimental results of benzaldehyde sorption are compared with pentanal in Fig. 9. Compared to the sorption of pentanal it is shown that the sorption of benzaldehyde is rather slow. Obtained reaction rate constants are listed in Table 2. It appears that they are an order of magnitude lower than those of pentanal. It is possible that this is due to the steric hindrances in case of benzaldehyde. Additionally, it is observed and explained by several authors (Cordes and Jencks 1962, 1963; Martin 1964) that the Schiff base formation mechanism is different for aromatic and aliphatic compounds. In general, this reaction is acid catalyzed, but in case of aromatics the acid catalyses the formation of the intermediate tetrahedral complex, whereas for aliphatics it catalyses the subsequent water elimination.

Unlike the sorption of pentanal, the sorption of benzaldehyde with impregnated resins is influenced by both reactive extraction and surface adsorption (Babić et al. 2008). Therefore it can not be assumed that the polymeric support is inert. However, it can be seen from Fig. 9 that the model fits the experimental data rather well. This suggests that the surface diffusion is negligible (Hoogendoorn et al. 1994; Prasher and Ma 1977).

\section{Conclusions}

The sorption kinetics of aldehydes from aqueous solutions with Primene JM-T impregnated Amberlite XAD-16 and MPP particles, was successfully investigated in a ZLC setup. A model to describe the sorption process in the EIR was developed in analogy to diffusion and reaction in a stagnant liquid sphere, but correcting for the porosity and particle properties influencing the diffusion. The developed model describes the kinetic behavior of the process in the low concentration region rather well. In the high concentration region the initial sorption rate is well predicted. Unfortunately, approaching the equilibrium the model starts deviating from the experimental data.

Particle morphology and the support material do influence the sorption rate. The effective diffusivity of aldehydes is faster in XAD-16 than in MPP impregnated particles. The reaction rate constants were determined by fitting the model to the experimental data. For both EIRs the same value of the reaction rate constant was obtained. The sorption of benzaldehyde is much slower than the sorption of pentanal due to the difference in the chemical reaction rate constant which is an order of magnitude lower for benzaldehyde $\left(\sim 10^{-4} \mathrm{l} / \mathrm{mol} \mathrm{s}\right)$ than for pentanal $\left(\sim 10^{-3} \mathrm{l} / \mathrm{mol} \mathrm{s}\right)$. This is probably due to the difference in reaction mechanism of aromatic and aliphatic Schiff base formation. The effect of initial aldehyde concentration, extractant loading and particle size on the sorption rate is well described by the model. Particle size effect is dominated by the diffusion time constant which is inversely proportional to $R^{2}$.

Open Access This article is distributed under the terms of the Creative Commons Attribution Noncommercial License which permits any noncommercial use, distribution, and reproduction in any medium, provided the original author(s) and source are credited.

\section{References}

Babić, K., van der Ham, L., de Haan, A.B.: Recovery of benzaldehyde from aqueous streams using extractant impregnated resins. React. Funct. Polym. 66(12), 1494-1505 (2006)

Babić, K., van der Ham, L., de Haan, A.B.: Removal of aldehydes from aqueous streams using extractant impregnated resins. AlChE J. (2008, submitted)

Bhandari, V.M., Juvekar, V.A., Pathwardhan, S.R.: Modified shrinking core model for reversible sorption on ion-exchange resins. Sep. Sci. Technol. 27, 1043-1064 (1992)

Cordes, E.H., Jencks, W.P.: On the mechanism of Schiff base formation and hydrolysis. J. Am. Chem. Soc. 84(5), 832-837 (1962)

Cordes, E.H., Jencks, W.P.: The mechanism of hydrolisis of schiff bases derived from aliphatic amines. J. Am. Chem. Soc. 85(18), 2843-2848 (1963)

Cortina, J.L., Warshawsky, A.: Developments in solid-liquid extraction by solvent-impregnated resins. In: Marinsky, J.A., Marcus, Y. (eds.) Ion Exchange and Solvent Extraction, vol. 13, pp. 195293. Dekker, New York (1997) 
Cortina, J.L., Arad-Yellin, R., Miralles, N., Sastre, A.M., Warshawsky, A.: Kinetics studies on heavy metal ions extraction by Amberlite XAD2 impregnated resins containing a bifunctional organophosphorous extractant. React. Funct. Polym. 38, 269-278 (1998)

Doraiswamy, L.K., Sharma, M.M.: Heterogeneous Reactions: Analysis, Examples and Reactor Design, 1st edn. Fluid-Fluid-Solid Reactions, vol. 2, pp. 17-45. Wiley, New York (1984)

Dunnewijk, J., Bosch, H., de Haan, A.B.: Adsorption kinetics of CoCl2 and $\mathrm{PPh} 3$ over macroreticular and gel type adsorbents by a generalized ZLC method. Chem. Eng. Sci. 61, 4813-4826 (2006)

Eić, M., Ruthven, D.M.: A new experimental technique for measurement of intercrystalline diffusivity. Zeolites 8, 40-45 (1988)

gPROMS ${ }^{\circledR}$ Advanced User Guide, Chap. 3, Process System Enterprise, London, UK (2007)

Hoogendoorn, J.A., Versteeg, G.F., van Swaaij, W.P.M.: Mass transfer accompanied by reversible chemical reactions in an inert porous sphere impregnated with a stagnant liquid. Chem. Eng. Sci. 48(15), 2727-2740 (1993)

Hoogendoorn, J.A., Versteeg, G.F., van Swaaij, W.P.M.: Experimental study of the absorption of acid gases in porous particles impregnated with aqueous alkanolamine solutions. Chem. Eng. Sci. 49(20), 3421-3438 (1994)

Juang, R.-S., Lin, H.-C.: Metal sorption with extractant-impregnated macroporous resins. 1. Particle diffusion kinetics. J. Chem. Tech. Biotechnol. 62, 132-140 (1995a)

Juang, R.-S., Lin, H.-C.: Metal sorption with extractant-impregnated macroporous resins. 2. Chemical reaction and particle diffusion kinetics. J. Chem. Tech. Biotechnol. 62, 141-147 (1995b)

Kabay, N., Arda, M., Saha, B., Streat, M.: Removal of Cr(VI) by solvent impregnated resins (SIR) containing Aliquat 336. React. Funct. Polym. 54, 103-115 (2003)

Komiyama, H., Smith, J.M.: Intraparticle mass transport in liquid-filled pores. AIChE J. 20(4), 728-734 (1974)

Kostova, A., Tsibranska, I., Bart, H.-J.: Study of phenylalanine sorption kinetics on solvent impregnated resins, part II. Solvent Extr. Ion Exch. 25(1), 127-145 (2007)

Lee, S.C.: Kinetics of reactive extraction of penicillin $\mathrm{G}$ by Amberlite LA-2 in kerosene. AIChe J. 50(1), 119-126 (2004)
Ma, Y.H., Evans, L.B.: Transient diffusion from a well stirred reservoir to a body of arbitrary shape. AIChE J. 14(6), 956-961 (1968)

Martin, B.: Reactions of carbonyl compounds with amines and derivatives. J. Phys. Chem. 68(6), 1369-1377 (1964)

Nikhade, B.P., Pangarkar, V.G.: Equilibria and kinetics of extraction of citric acid from aqueous solutions in Alamine 336-cyclohexanone system. Sep. Sci. Technol. 40(12), 2539-2554 (2005)

Prasher, B.D., Ma, Y.H.: Liquid diffusion in microporous alumina pellets. AIChe J. 23(3), 303-312 (1977)

Reid, R.C., Prausnitz, J.M., Poling, B.E.: The Properties of Gases and Liquids. McGraw-Hill International Editions (1988)

Ruiz, M.O., Cabezas, J.L., Escudero, I., Coca, J.: $\alpha$-Phenylglycine extraction with a trialkylmethylammonium chloride-impregnated macroporous resin. 2. Kinetics. Trans. IChemE 80(A), 537-542 (2002)

Ruiz, M.O., Cabezas, J.L., Escudero, I., Coca, J.: Valeric acid extraction with tri-n-butyl prosphate impregnated in a macroporous resin. I. Equilibrium and mass transfer rates. Sep. Sci. Technol. 39(1), 79-95 (2004)

Ruthven, M.D., Stapleton, P.: Measurement of liquid phase counterdiffusion in zeolites by the ZLC method. Chem. Eng. Sci. 48, 8998 (1993)

Saha, B., Gill, R.J., Bailey, D.G., Kabay, N., Arda, M.: Sorption of $\mathrm{Cr}(\mathrm{VI})$ from aqueous solution by Amberlite XAD-7 resin impregnated with Aliquat 336. React. Funct. Polym. 60, 223-244 (2004)

Serarols, J., Poch, J., Llop, M.F., Villaescusa, I.: Determination of the effective diffusion coefficient for gold(III) on a macroporous resin XAD-2 impregnated with triisobutyl phosphine sulfide. React. Funct. Polym. 41, 27-35 (1999)

Serarols, J., Poch, J., Villaescusa, I.: Determination of the effective diffusion coefficient of $\mathrm{Zn}(\mathrm{II})$ on a macroporous resin XAD-2 impregnated with di-2-ethylhexyl phosphoric acid (DEHPA). Influence of metal concentration and particle size. React. Funct. Polym. 48, 53-63 (2001)

Traving, M., Bart, H.-J.: Recovery of organic acids using ionexchanger-impregnated resins. Chem. Eng. Technol. 25(10), $997-$ $1003(2002)$ 